\title{
Screening and identification of significant genes related to tumor metastasis and PSMA in prostate cancer using microarray analysis
}

\author{
LIN XU ${ }^{1-3 *}$, ZHU WANG $^{1-3 *}$, XIAO-FEI LI ${ }^{4}$, XIA HE $^{1-3}$, LIN-LIN GUAN $^{1-3}$, JIU-LING TUO $^{1-3}$, \\ YANG WANG $^{1-3}$, YANFEN LUO ${ }^{1-3}$, HUI-LING ZHONG ${ }^{1-3}$, SHAO-PENG QIU ${ }^{4}$ and KAI-YUAN CAO ${ }^{1-3}$ \\ ${ }^{1}$ Department of Microbiology, Zhongshan School of Medicine, ${ }^{2}$ Key Laboratory of Tropical Disease Control, \\ Ministry of Education, ${ }^{3}$ Research Center for Clinical Laboratory Standard, Zhongshan School of Medicine, \\ ${ }^{4}$ Department of Urology, The First Affiliated Hospital, Sun Yat-Sen University, Guangzhou 510080, P.R. China
}

Received June 22, 2013; Accepted July 18, 2013

DOI: $10.3892 / o r .2013 .2656$

\begin{abstract}
Tumor metastasis is one of the causes for the high mortality rate of prostate cancer $(\mathrm{PCa})$ patients, yet the molecular mechanisms of PCa metastasis are not fully understood. In our previous studies, we found that PSMA suppresses the metastasis of $\mathrm{PCa}$, yet the underlying mechanism remains unknown. To identify the genes related to tumor metastasis possibly regulated by PSMA, we performed tumor metastasis PCR array assay to analyze the differentially expressed tumor metastasis-related genes. Eighty-four tumor metastasis related genes were screened in si-PSMA LNCap cells (PSMA silenced by siRNA)/LNCap cells and in PC-3/ LNCap cells, respectively. Expression levels of possible related genes were verified by real-time PCR in 4 prostate cancer cell lines (LNCap, 22RV1, PC-3 and DU145) and in 85 clinical samples (12 normal, 26 benign prostatic hypertrophy and 47 prostate cancer tissues). The results showed that 10 genes (including CDH6 and CXCL12) were upregulated and 4 genes (CCL7, ITGB3, MDM2 and MMP2) were downregulated in the si-PSMA LNCap cells. There were 41 genes significantly upregulated and 15 genes downregulated in PC-3 cells when compared with LNCap cells. Eight common genes were found in both the si-PSMA and PSMA(-) groups. CDH6, MMP3, MTSS1 were further identified as PSMA-related genes in the prostate cancer cell lines and clinical samples, and their expression showed a negative correlation with the stage of prostate cancer $(\mathrm{P}<0.0001)$ and PSMA level $(\mathrm{P}<0.05)$ in clinical samples, indicating their possible involvement in PSMA-related PCa metastasis regulation. These findings may provide insights into the mechanism involved in the suppres-
\end{abstract}

Correspondence to: Professor Kai-Yuan Cao, Research Center for Clinical Laboratory Standard, Zhongshan School of Medicine, Sun Yat-sen University, Guangzhou 510080, P.R. China

E-mail: caoky@mail.sysu.edu.cn

${ }^{*}$ Contributed equally

Key words: prostate cancer, prostate-specific membrane antigen, tumor metastasis, microarray analysis sion of PCa metastasis by PSMA and its possible interacting proteins, and may provide clues for further exploration of the molecular mechanism of PCa metastasis.

\section{Introduction}

Prostate cancer ( $\mathrm{PCa}$ ) is one of the most common malignant tumors in males and the fifth leading cause of cancer-related mortality worldwide, and the second leading cause in the male population $(1,2)$. Because the onset of $\mathrm{PCa}$ is insidious, early diagnosis is difficult, and many patients have metastatic disease at the time of diagnosis $(3,4)$ and death from $\mathrm{PCa}$ occurs largely in patients with aggressive androgen-insensitive metastatic disease (5). Upon sustained hormone-ablation therapy, invasive PCa frequently acquires castration-resistant features and gains metastatic potential, which accounts for high patient morbidity (6). Even though initially certain PCa cases are androgen-dependent (ADPC), eventually, if sufficient time is allowed to elapse, virtually all prostate cancers become androgen-independent (AIPC). The mechanisms leading to androgen independence have been extensively studied, yet the mechanisms are still not fully understood $(7,8)$.

Prostate-specific membrane antigen (PSMA), a transmembrane-carboxypeptidase $(9,10)$, as a prostate cancer-specific biomarker and potential target for diagnosis and treatment, has been correlated with aggressive disease and has obtained wide attention $(1,11,12)$. It has been confirmed that PSMA is $\mathrm{PCa}$-specific and is highly expressed in PCa. Its expression is further increased in poorly differentiated, advanced, hormonerefractory metastatic $\mathrm{PCa}$, therefore, it is an ideal marker for prostate cancer diagnosis and a target for treatment $(13,14)$. The high expression of PSMA in PCa and its correlation with pathological classification and prognosis indicate that PSMA may play a regulatory role in PCa development and metastasis.

Although a high level of PSMA is usually associated with PCa progression, the role of PSMA in tumor migration and invasiveness is still uncertain, and contradictory results exist (15-17). A recent study by Ghosh et al (17) revealed that PSMA suppresses PC-3 cell invasiveness. Previous studies by our group found that PSMA and its spliced variant are highly expressed in PCa tissues (11), suppress prostate cancer cell proliferation, migration and invasiveness, and blockage of 
Table I. Pathological information regarding the prostate samples used in real-time PCR identification of the MMP3, MTSS1 and CDH6 genes.

\begin{tabular}{lcc}
\hline Tissue type & $\begin{array}{c}\text { Gleason } \\
\text { score }\end{array}$ & $\begin{array}{c}\text { No. of tissue } \\
\text { samples } \\
\text { analyzed }\end{array}$ \\
\hline Normal prostate & & 12 \\
Benign prostatic hypertrophy (BPH) & $4,5,6$ & 26 \\
Primary prostate cancer & $7,8,9$ & 30 \\
Primary prostate cancer & 17 \\
\hline
\end{tabular}

Totally, 85 clinical samples were collected for real-time PCR identification of the MMP3, MTSS1 and CDH6 genes. Forty-seven prostate cancer specimens were analyzed and divided into two categories according to the Gleason score value.

PSMA in LNCap cells promotes the invasion and migration of prostate cancer cells (12). Yet, the mechanism of PSMA involved in the process of metastasis of PCa remains unclear. In the present study, in order to screen and identify metastasisrelated genes that are possibly involved in PCa metastasis regulated by PSMA, we used a tumor metastasis PCR array to analyze the expression of 84 tumor metastasis-related genes in LNCap cells (a known PSMA-positive prostate cancer cell line), si-PSMA LNCap cells and PC-3 cells (known prostate cancer cell line that does not express PSMA), respectively, and we compared the common differentially expressed genes to screen the possible PSMA-related genes. Three of these candidate genes were further confirmed in prostate cancer cell lines and also in clinical prostate samples. Our findings may provide clues for exploring the role of PSMA in PCa metastasis and its molecular mechanism.

\section{Materials and methods}

Cell lines, tissue samples and reagents. Prostate cancer cell lines LNCap, 22RV1, PC-3 and DU145 were obtained from the ATCC (American Type Culture Collection, Manassas, VA, USA) and cultured in RPMI-1640 medium (Gibco-BRL, Invitrogen Corp., Grand Island, NY, USA) with $1 \mathrm{mM}$ sodium pyruvate, $100 \mathrm{U} / \mathrm{ml}$ penicillin $\mathrm{G}, 100 \mu \mathrm{g} / \mathrm{ml}$ streptomycin (Sigma Corp. of America, Ronkonkoma, NY, USA), 10\% fetal bovine serum (FBS; Tianjin Hao Yang Biological Manufacture Co., Ltd., Beijing, China). Lipofectamine ${ }^{\mathrm{TM}} 2000$ transfection reagent, TRIzol and the One-Step Fluorescence assay kit were products of Invitrogen (Carlsbad, CA, USA).

Prostate tissue samples including normal prostate $(n=12)$, primary prostate cancer $(n=47)$, and benign prostatic hypertrophy $(\mathrm{BPH})(\mathrm{n}=26)$, were obtained at the Department of Urology, the First Affiliated Hospital of Sun Yat-sen University, Guangzhou, China. All tissue samples were stored at $-80^{\circ} \mathrm{C}$ until being further processed. The Gleason score was used to evaluate tumor malignancy, and the prostate cancer samples were then divided into two categories according to their Gleason score (Table I). Informed content for the scientific use of biologic materials was obtained from all patients in accordance with the requirements of the Medical Ethics Review Board of Sun Yat-sen University.

Reverse transcription and real-time PCR. One step TaqMan real-time RT-PCR was used to evaluate the PSMA silencing effect. Specific probes and primers of PSMA were designed by Beacon Designer 7.01 Demo, and the $\beta$-actin gene served as the internal control. Primer and probe sequences are shown in Table II. One step real-time PCR amplification reaction of PSMA contained $1 \mu \mathrm{l}$ SuperScript III RT/Platinum Taq Mix, $1 \mu \mathrm{l}$ RNaseOut, $25 \mu \mathrm{l} 2 \mathrm{X}$ reaction mixture (Invitrogen), $0.5 \mu \mathrm{M}$ of the forward and reverse primers and $0.5 \mu \mathrm{M}$ of the probe, and $3 \mu \mathrm{l}$ of RNA and nuclease-free water to a final volume of $50 \mu 1$. Real-time RT-PCR was performed on an ABI 7500 realtime PCR system (Applied Biosystems, Foster City, CA, USA) using thermal cycling conditions as follows: $50^{\circ} \mathrm{C}$ for $15 \mathrm{~min}$, $95^{\circ} \mathrm{C}$ for $2 \mathrm{~min} ; 95^{\circ} \mathrm{C}$ for $15 \mathrm{sec}, 60^{\circ} \mathrm{C}$ for $30 \mathrm{sec}, 40$ cycles. All PCR reactions were performed in duplicate.

Reverse transcription (RT) and real-time PCR based on SYBR-Green detection was used to identify candidate PSMArelated genes in PCa cell lines and tissues. Total RNA was extracted by TRIzol reagent following the manufacturer's instructions. Briefly, PCa cell total RNA was extracted directly in a $3.5 \mathrm{~cm}$ diameter culture dish by adding $1 \mathrm{ml}$ TRIzol reagent, and the total RNA of the PCa clinical samples that were cut into $\sim 0.5 \mathrm{~cm}$ pieces, adding the appropriate volume of pre-cooling TRIzol regeant at v/v 10:1. RT reaction was carried out in a final volume of $20 \mu \mathrm{l}$ using the Invitrogen reverse transcription kit (Invitrogen) according to the manufacturer's instructions. First, the reaction mixture containing $500 \mathrm{ng} / \mu \mathrm{l}$ Oligo $(\mathrm{dT})_{18}(1 \mu \mathrm{l})$, total RNA $(1.5 \mu \mathrm{g}), 10 \mathrm{mM}$ each deoxynucleoside triphosphate (dNTP) $(1 \mu \mathrm{l})$ and nuclease-free water up to a volume of $13 \mu \mathrm{l}$ was incubated for $5 \mathrm{~min}$ at $65^{\circ} \mathrm{C}$, placed on ice for more than $1 \mathrm{~min}$, then $5 \mathrm{X}$ first strand buffer (4 $\mu \mathrm{l}), 0.1 \mathrm{M}$ DTT $(1 \mu \mathrm{l}), \mathrm{RNase}$ inhibitor $(1 \mu \mathrm{l}), 200 \mathrm{U} / \mu \mathrm{l}$ SuperScript III reverse transcriptase $(1 \mu \mathrm{l})$ were added to a final reaction volume of $20 \mu \mathrm{l}$. The thermal cycling parameters for the RT reaction were as follows: $60 \mathrm{~min}$ at $50^{\circ} \mathrm{C}$ and $15 \mathrm{~min}$ at $70^{\circ} \mathrm{C}$. Products of RT reactions were stored at $-20^{\circ} \mathrm{C}$ until further analysis. The mRNA expression of candidate genes was determined using the ABI PRISM 7500 detection system (Applied Biosystems) and SYBR Premix Taq ${ }^{\mathrm{TM}}$ (Invitrogen). The primer sequences are shown in Table II. The reaction system contained 2X SYBR Premix Ex Taq ${ }^{\mathrm{TM}}$ II $(10 \mu \mathrm{l})$, $0.4 \mu \mathrm{M}$ of each forward and reverse primers, 50X ROX reference dye $0.4 \mu \mathrm{l}$ and $2 \mu \mathrm{l}$ of the synthesized cDNA in a $20 \mu \mathrm{l}$ reaction volume. Thermal cycling conditions were as follows: $95^{\circ} \mathrm{C}$ for $30 \mathrm{sec}$, followed by 40 cycles of $95^{\circ} \mathrm{C}$ for $5 \mathrm{sec}, 60^{\circ} \mathrm{C}$ for $30 \mathrm{sec}$. All PCR reactions were performed in duplicate and repeated three times. Melting curve analysis was used to control the amplification specificity. Target gene expression is shown as the fold increase or decrease relative to the expression of $\beta$-actin. Fold-changes in target gene mRNA expression were determined as $2^{-\Delta \Delta \mathrm{Ct}}$ using the same calculation formula as determined in the microarray analysis.

PSMA gene silencing by siRNA in LNCap cells. Three pairs of siRNAs for PSMA (GenBank ID: M99487) were designed by online software (http://jura.wi.mit.edu/bioc/siRNAext/ siRNA_search.cgi?tasto=10925811) (Table III). Designed 
Table II. Real-time PCR primer and probe sequences.

\begin{tabular}{lll}
\hline & Primer/Taq & \multicolumn{1}{c}{ Primer and TaqMan probe sequences } \\
\hline TaqMan real-time PCR & PSMA-F & 5'-GAAGTCTCAAAGTGCCCTACAATG-3' \\
& PSMA-R & 5'-ACCCATGAGTCCCGGTGAC-3' \\
PSMA-Taq & 5'-(FAM) CTGTCTGGTTCCACTGCTCCTCTGA (Eclipse)-3' \\
Actin-F & 5'-GACTACCTCATGAAGATCCTCACC-3' \\
Actin-R & 5'-TCTCCTTAATGTCACGCACGATT-3' \\
Actin-Taq & 5'-(FAM) CGGCTACAGCTTCACCACCACGGC (Eclipse)-3' \\
SYBR-Green-based real-time PCR & PSMA-F & 5'-ACACCGCTGATGTACAGCTTGGT-3' \\
& PSMA-R & 5'-TGCTTATCCTGGGCATGCCACTG-3' \\
& MMP3-F & 5'-GGCAGGCAAGACAGCAAGGCAT-3' \\
& MMP3-R & 5'-GCTGAGCAAACTGCCACGCAC-3' \\
& MTSS1-F & 5'-GCGAGAACCGGACCCCAACG-3' \\
& MTSS1-R & 5'-AGGCCTGGTGGCAGCCGATA-3' \\
& CDH6-F & 5'-TTTTGGGTGGGCCAGCCCTACC-3' \\
& CDH6-R & 5'-CAGAGAGCTCCAGGGCCCTTTTCT-3' \\
& Actin-F & 5'-CAGAGCCTCGCCTTTGCCGATCC-3' \\
& Actin-R & 5'-CCTTGCACATGCCGGAGCCGT-3'
\end{tabular}

TaqMan real-time RT-PCR was used to evaluate the PSMA silencing effect on LNCap cells transfected with PSMA-siRNA. SYBR-Greenbased real-time PCR was used to identify candidate PSMA-related genes in prostate cancer cell lines and tissues. The primers and probes used in real-time PCR are shown. F, forward primer; R, reverse primer; Taq, TaqMan probe.

Table III. siRNA sequences targeting the PSMA gene.

\begin{tabular}{llc}
\hline siRNA duplex & \multicolumn{1}{c}{ Nucleotide sequences } & Position in the PSMA gene coding sequence $^{\mathrm{a}}$ \\
\hline siRNA-1 & $\begin{array}{l}\text { Sense 5'-CUGAGAACAUCAAGAAGUUdTdT-3' } \\
\text { Antisense 3'-dTdTGACUCUUGUAGUUCUUCAA-5' }\end{array}$ & $461-479$ \\
siRNA-2 & $\begin{array}{l}\text { Sense 5'-GGCAAAUCUCUUUAUGAAAdTdT-3' } \\
\text { Antisense 3'-dTdTCCGUUUAGAGAAAUACUUU-5' }\end{array}$ & $1729-1747$ \\
siRNA-3 & $\begin{array}{l}\text { Sense 5'-GCGAUCUAGUGUAUGUUAAdTdT-3' } \\
\text { Antisense 3'-dTdTCGCUAGAUCACAUACAAUU-5' }\end{array}$ & $776-794$ \\
\hline Three pairs of PSMA siRNAs of PSMA were designed by online software (see Materials and methods). . Position according to the sequence of \\
PSMA (GenBank ID: M99487).
\end{tabular}

siRNAs and the positive and negative control siRNAs were synthesized and supplied by Guangzhou RiboBio Co., Ltd. (Guangzhou, China). The day before transfection, LNCap cells were collected, digested with $0.25 \%$ trypsin and counted, and then seeded onto 6-well plates (Corning Incorporated Costar, Corning, NY, USA) at the initial density of $2 \times 10^{5}$ cells/well and cultured with antibiotic-free medium. The transfection was performed by Lipofectamine ${ }^{\mathrm{TM}} 2000$ with the concentration of siRNA at $50 \mathrm{nM}$, and LNCap cells transfected with the negative control siRNA (supplemented by RiboBio) served as the control. Total protein and total RNA were extracted $72 \mathrm{~h}$ after transfection. One step real-time RT-PCR was used to evaluate the PSMA silencing effect at the mRNA level. Silencing effect of PSMA at the protein level was identified by western blotting. The monoclonal antibody (YPSMA, ab19071; Abcam Inc., Cambridge, MA, USA) used in the western blotting recognized 716-723 amino acid residues of the $\mathrm{C}$ terminus of PSMA. The antibody dilution ratio used in the western blotting was 1:1,000.

Microarray analysis. Human tumor metastasis PCR array (SABiosciences, Qiagen, Gaithersburg, MD, USA) was designed to analyze 96 genes by real-time PCR, including 84 genes known to be involved in tumor metastasis, 4 housekeeping genes (HK genes) and 8 quality control genes. Tumor metastasis-related genes selected for this array encode several classes of protein factors including cell adhesion, ECM components, cell cycle, cell growth and proliferation, apoptosis, transcription factors and regulators and other genes related to tumor metastasis.

Two groups were designed for the tumor metastasis PCR array analysis: the PSMA(-) group was used to analyze the 
differentially expressed genes between the LNCap and PC-3 cells, and the si-PSMA group was used to analyze the differentially expressed genes between the si-PSMA LNCap and LNCap cells. The mean value of the replicates for each group was calculated and expressed as the cycle threshold $(\mathrm{Ct})$. The amount of gene expression was then calculated as the difference $(\Delta \mathrm{Ct})$ between the $\mathrm{Ct}$ value obtained for the target gene and the $\mathrm{Ct}$ value obtained for the $\mathrm{HK}$ genes. The value of $\Delta \mathrm{Ct}$ was calculated for each pathway-focused gene in each group using the following calculation formula: $\Delta \mathrm{Ct}$ (control group) $=$ average $\mathrm{Ct}$ of the target gene - average $\mathrm{Ct}$ of the $\mathrm{HK}$ genes for the control group array; $\Delta \mathrm{Ct}$ (experimental group) $=$ average $\mathrm{Ct}$ of the target gene - average $\mathrm{Ct}$ of the $\mathrm{HK}$ genes for the experimental group array. The $\Delta \Delta \mathrm{Ct}$ was calculated for each gene across two PCR arrays (or groups), $\Delta \Delta \mathrm{Ct}=\Delta \mathrm{Ct}$ (experimental group) - $\Delta \mathrm{Ct}$ (control group). The fold-change for each gene from the control group to the experimental group was calculated as $2^{-\Delta \Delta \mathrm{Ct}}$.

Candidate gene identification in the PCa cell lines and tissues. Based on the comparison of the common genes between the PSMA(-) group and the si-PSMA group, and the previous studies and reports, the MMP3, MTSS1 and CDH6 genes were chosen and further analyzed by reverse transcription (RT) and SYBR-Green-based real-time PCR in PCa cell lines including the PSMA-positive cell lines (LNCap and 22RV1) and the PSMA-negative cell lines (PC-3 and DU145), and also 85 clinical samples of prostate tissues, including 12 normal prostate, $26 \mathrm{BPH}$ and 47 prostate cancer samples. The pathological information regarding the prostate tissues is shown in Table I.

Statistical analysis. The PSMA mRNA expression levels were not distributed normally, therefore the log-transformed relative expression levels were used to obtain sufficiently normally distributed values. The differences in expression of MMP3, MTSS1 and CDH6 genes among normal prostate, BPH and PCa were evaluated using one-way ANOVA. Spearman correlation coefficient was used to evaluate the correlation between Gleason score and the levels of MMP3, MTSS1, CDH6 and PSMA mRNA expression, and also between PSMA and MMP3, MTSS1 and CDH6. P-values $<0.05$ were considered to indicate a statistically significant result. All statistical analyses were performed using SPSS11.5 software (SPSS Inc., Chicago, IL, USA).

\section{Results}

Construction of si-PSMA LNCap cells. Stable PSMA silenced LNCap cell lines (si-PSMA LNCap cells) were successfully obtained by transfection with the siRNA sequences. Seventy-two hours after transfection with the siRNAs, the PSMA gene expression level was detected by western blotting and real-time PCR. Real-time PCR showed that the PSMA mRNA expression level was downregulated $>4$-fold by siRNA-3, and the western blot analysis showed that the PSMA protein expression level was reduced $68 \%$ by siRNA-3 (Chi-square test, $\mathrm{P}<0.01$ compared to the negative control; Fig. 1). siRNA-1 and siRNA-2 exhibited a weaker silencing effect than siRNA-3, therefore, siRNA-3 was chosen for further study.
Screening of differentially expressed tumor metastasis-related genes between LNCap and PC-3 cells by tumor metastasis $P C R$ array. In order to gain better understanding of the molecular mechanism involved in the metastatic progression of androgen-independent prostate cancer cells, we performed a comprehensive metastasis-related gene expression profile analysis of PSMA-positive LNCap and PSMA-negative PC-3 cells, namely the PSMA(-) group, using a tumor metastasis PCR array. Genes whose expression was upregulated or downregulated $>2$-fold, were considered significantly differentially expressed and were selected for further analysis. Forty-one genes had significantly higher expression in the PC-3 cells $(\mathrm{P}<0.05$; Fig. 2), among which 23 genes were expressed $>10$-fold (Table IV). The genes with the highest upregulated expression in the PC-3 cells were CDH11 (87,443.2-fold). There were 15 genes with downregulated expression in the PC-3 cells when compared to the LNCap cells; among which the expression levels of 6 genes (TP53, PTEN, CTNNA1, CDH1, MDM2 and IGF1) were $>10$-fold (Table IV). Most of the significant genes identified in the present study have not been previously reported in prostate cancer.

Screening of differentially expressed tumor metastasisrelated genes between LNCap and si-PSMA LNCap cells by the tumor metastasis PCR array. We further analyzed the differential expression of tumor metastasis-related genes in the si-PSMA LNCap cells in which PSMA expression was blocked. Tumor metastasis PCR array analysis revealed that 10 genes were upregulated (namely CDH6, CXCL12, IL18, IL8RB, IGF1, MMP13, MMP3, MTSS1, TSHR and ITGA7) and 4 genes were downregulated (CCL7, ITGB3, MDM2 and MMP2) significantly in the si-PSMA LNCap cells when compared to the PSMA non-silenced LNCap cells. The differentially expressed genes and their description are documented in Table V. Consistent with other reports, the IGF1 gene has been previously reported to be upregulated in C4-2 cells when compared with LNCap cells (18). Among the total 14 genes that were significantly altered in the si-PSMA LNCap cells, the MDM2 gene showed the maximum downregulated change when PSMA expression was suppressed (Table V), consistent with a previous report (19).

Screening of tumor metastasis-related genes possibly related to PSMA by tumor metastasis PCR array. By comparison of the differentially expressed genes between the PSMA(-) group and the si-PSMA group, we aimed to identify those common genes that may be regulated by or related to PSMA. From the tumor metastasis PCR array results, we found that expression of 6 genes (namely IL18, MTSS1, MMP13, ITGA7, MMP3 and CDH6) was significantly upregulated in both the si-PSMA LNCap and the PC-3 cells, although their expression levels were different. Expression of 2 genes (MDM2 and MMP2) was downregulated to a similar extent in both the PC-3 and the si-PSMA LNCap cells. The results indicate that these 8 genes may be related to the role of PSMA in tumor metastasis.

The genes differently expressed between the PSMA(-) and si-PSMA groups included 2 genes (CCL7 and ITGB3) that were upregulated significantly in the si-PSMA LNCap cells but not in the PC-3 cells. Expression of 13 genes was significantly lower in the PC-3 cells but not in the si-PSMA LNCap 

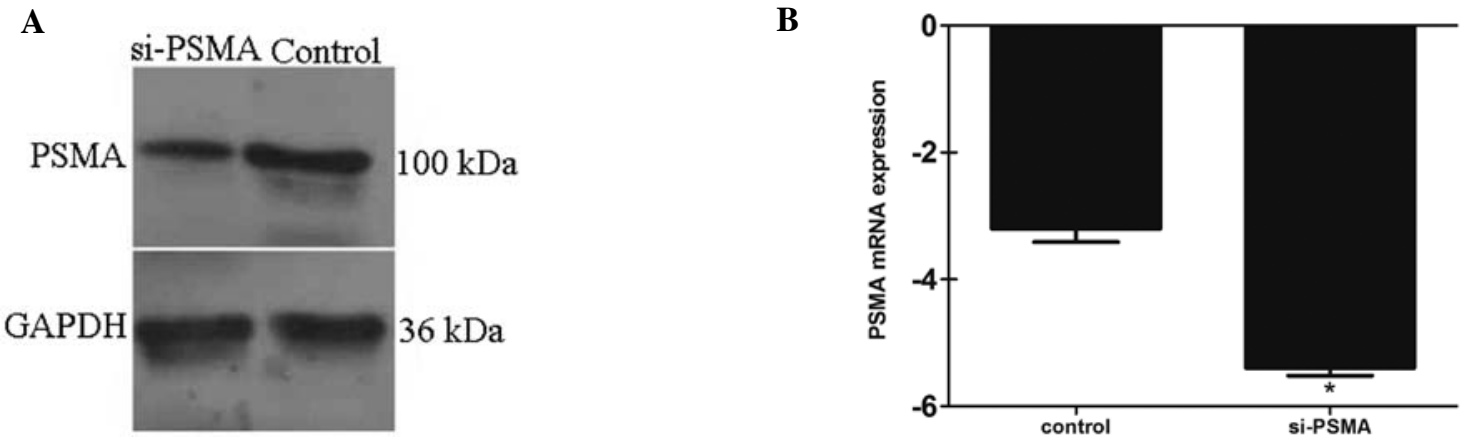

Figure 1. Western blotting and qPCR assay of the PSMA protein and mRNA silencing effect. Designed siRNA-3 was transfected into LNCap cells using Lipofectamine 2000. The silencing effect was confirmed by real-time PCR and western blotting. (A) Western blot results of the PSMA silencing effect induced by siRNA-3. si-PSMA, LNCap cells transfected using siRNA-3; Control, LNCap cells transfected with a negative control siRNA. (B) Real-time PCR results of the PSMA silencing effect induced by siRNA-3. Expression levels were normalized to $\beta$-actin expression. The error bars represent standard deviation (SD) values calculated from three parallel experiments. ${ }^{*} \mathrm{P}<0.01$.

Table IV. Tumor metastasis-related genes upregulated or downregulated $>10$-fold in PC-3 cells compared with LNCap cells.

Gene

Description

Fold-change: upregulation or

CDH11

Cadherin 11, type 2, OB-cadherin (osteoblast) downregulation

CD44

CD44 molecule (Indian blood group)

IL1B

Interleukin $1, \beta$

$94,50.01$

IL18

Interleukin 18 (interferon- $\gamma$-inducing factor)

$16,76.25$

TGF $\beta$

Transforming growth factor, $\beta 1$

407.47

MET

Met proto-oncogene (hepatocyte growth factor receptor)

292.18

HGF

Hepatocyte growth factor (hepapoietin A; scatter factor)

290.7

MTSS1

Metastasis suppressor 1

142.19

CDH6

Cadherin 6, type 2, K-cadherin (fetal kidney)

112.47

FXYD5

FXYD domain containing ion transport regulator 5

82.12

71.28

PLAUR Plasminogen activator, urokinase receptor

54.97

MMP9

Matrix metallopeptidase 9 (gelatinase B, 92 kDa gelatinase, $92 \mathrm{kDa}$ type IV collagenase)

54.91

TIMP4

TIMP metallopeptidase inhibitor 4

50.48

ITGA7

Integrin, $\alpha 7$

35.24

SYK

Spleen tyrosine kinase

32.42

MCAM

Melanoma cell adhesion molecule

28.16

FN1

Fibronectin 1

NME4

Non-metastatic cells 4, protein expressed in

24.95

MMP13

Matrix metallopeptidase 13 (collagenase 3)

24.72

ETV4

Ets variant 4

TNFSF10

Tumor necrosis factor (ligand) superfamily, member 10

13.78

CDKN2A

Cyclin-dependent kinase inhibitor 2A (melanoma, p16, inhibits CDK4)

13.06

COL4A2

Collagen, type IV, $\alpha 2$

11.86

TP53

Tumor protein $\mathrm{p} 53$

$-22.72$

PTEN

Phosphatase and tensin homolog

$-67.89$

CTNNA1

Catenin (cadherin-associated protein), $\alpha 1,102 \mathrm{kDa}$

$-77.89$

CDH1

Cadherin 1, type 1, E-cadherin (epithelial)

$-195.5$

MDM2

MDM2 p53 binding protein homolog (mouse)

$-204.1$

IGF1

Insulin-like growth factor 1 (somatomedin C)

$-3,806$

Tumor metastasis PCR array analysis indicated that 23 genes were upregulated $>10$-fold in the PSMA-negative PC- 3 cells when compared with the PSMA-positive LNCap cells. Eight genes were upregulated >100-fold in PC- 3 cells; 6 genes were downregulated $>10$-fold in PC- 3 cells compared with LNCap cells. 

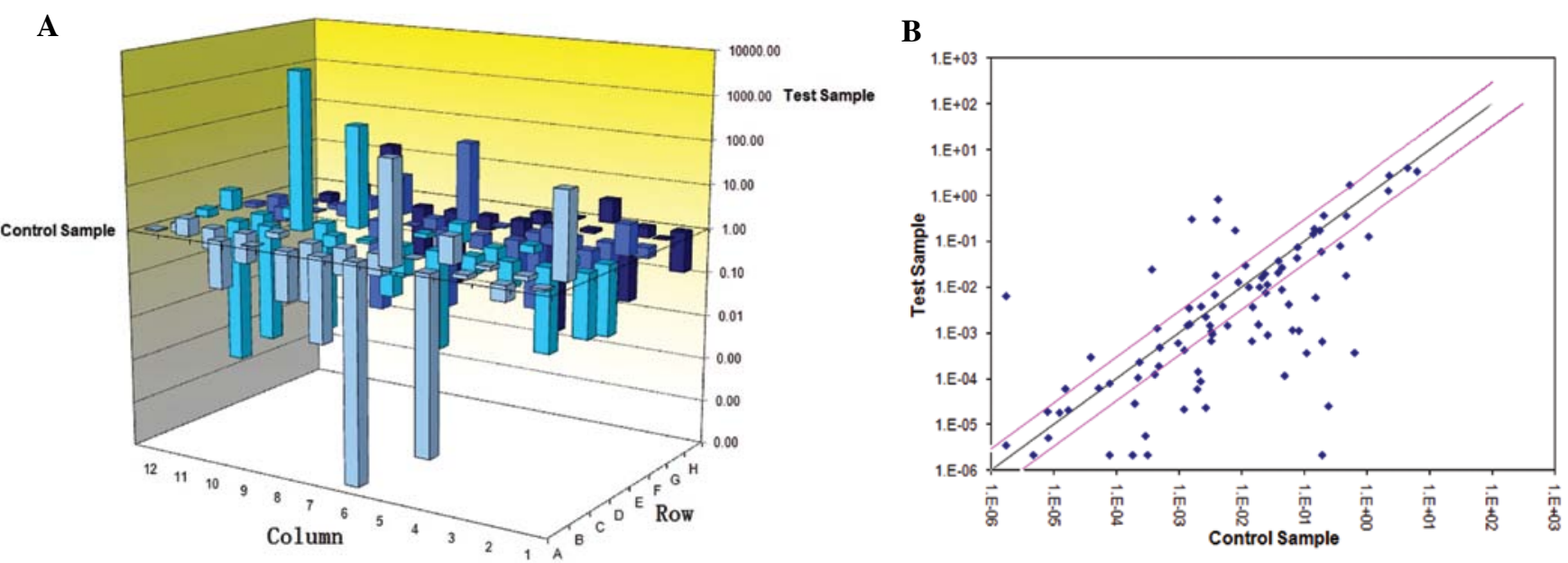

Figure 2. Tumor metastasis-related gene expression levels in the LNCap cells screened by human tumor metastasis PCR array. (A) 3D column chart results. The column indicates the expression ratio for each gene upregulated or downregulated in the LNCap cell sample. (B) Scatter chart results. The black line indicates fold-changes $\left(2^{-\Delta \Delta C t}\right)$ of 1 . The pink lines indicate the anticipated fold-change in gene expression threshold of 2. Genes whose expression was upregulated or downregulated $>2$-fold, were considered significantly differentially expressed.

Table V. Tumor metastasis-related genes upregulated or downregulated in the si-PSMA LNCap cells.

Gene

Description

Fold-change: upregulation or

$\mathrm{CDH} 6$ Cadherin 6, type 2, K-cadherin (fetal kidney) downregulation

\begin{tabular}{llr}
\hline CDH6 & Cadherin 6, type 2, K-cadherin (fetal kidney) & 2.88 \\
CXCL12 & Chemokine (C-X-C motif) ligand 12 (stromal cell-derived factor 1) & 2.51 \\
IGF1 & Insulin-like growth factor 1 (somatomedin C) & 2.04 \\
IL18 & Interleukin 18 (interferon- $\gamma$-inducing factor) & 2.04 \\
IL8RB & Interleukin 8 receptor, $\beta$ & 2.22 \\
ITGA7 & Integrin, $\alpha$ 7 & 2.25 \\
MMP13 & Matrix metallopeptidase 13 (collagenase 3) & 2.24 \\
MMP3 & Matrix metallopeptidase 3 (stromelysin 1, progelatinase) & 2.40 \\
MTSS1 & Metastasis suppressor 1 & 2.05 \\
TSHR & Thyroid stimulating hormone receptor & 2.82 \\
CCL7 & Chemokine (C-C motif) ligand 7 & -2.23 \\
ITGB3 & Integrin, $\beta 3$ (platelet glycoprotein IIIa, antigen CD61) & -2.05 \\
MDM2 & MDM2 p53 binding protein homolog (mouse) & -80.35 \\
MMP2 & Matrix metallopeptidase 2 (gelatinase A, 72 kDa gelatinase, 72 kDa type IV collagenase) & -2.29 \\
\hline
\end{tabular}

Tumor metastasis PCR array analysis indicated that 10 tumor metastasis-related genes were upregulated and 4 genes were downregulated in the si-PSMA LNCap cells when compared to the LNCap cell control. Genes whose expression level was upregulated or downregulated $>2$ fold were considered significantly differentially expressed.

cells. Moreover, expression of IGF1 and CXCL12 was significantly upregulated in si-PSMA LNCap cells, but significantly downregulated in the PC- 3 cells. The $\Delta \mathrm{Ct}$ changes in 28 genes were $<2$ and showed no significant change in both the PC-3 cells and the si-PSMA LNCap cells.

Verification of tumor metastasis-related gene expression in prostate cancer cell lines and clinical prostate samples. Three candidate genes, namely MMP3, CDH6 and MTSS1, were chosen for further analysis and identification in the PCa cell lines and prostate tissues by real-time RT-PCR. PCa cell lines used for verification included the PSMA-positive LNCap and
22RV1 cells, and the PSMA-negative PC-3 and DU145 cells. Consistent with the results of the tumor metastasis PCR array analysis based on LNCap and PC-3 cells, the MMP3 mRNA expression level was downregulated in the PSMA-positive cells including LNCap and 22RV1, and upregulated in the PSMA-negative cells including PC-3 and DU145 ( $\mathrm{P}<0.05$; Fig. 3A). CDH6 and MTSS1 levels were significantly higher in the PC-3 cells when compared with the other 3 cell lines $(\mathrm{P}<0.05$; Fig. 3A). Real-time PCR confirmed the microarray results for MMP3, CDH6 and MTSS1 in the LNCap and PC-3 cells, and the PSMA-positive 22RV1 cells showed the same differential expression trend as that in LNCap cells. In 


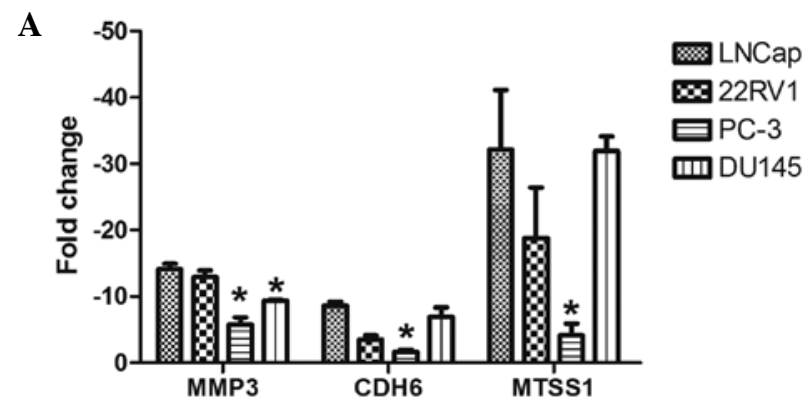

B
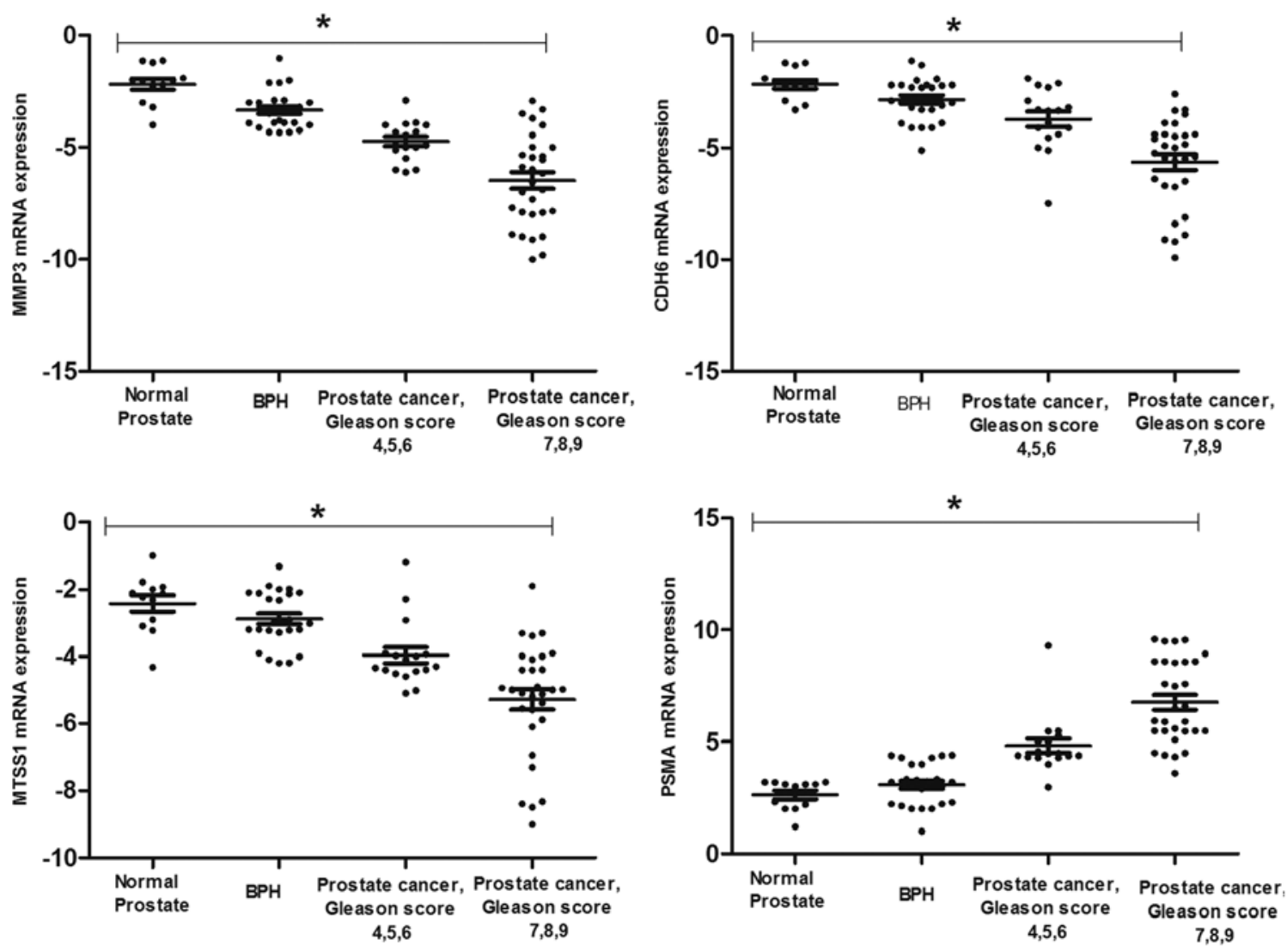

Figure 3. Identification of MMP3, MTSS1 and CDH6 gene expression in prostate cancer cell lines and clinical samples by real-time RT-PCR. PSMA-positive LNCap and 22RV1 cells, and PSMA-negative PC-3 and DU145 cells were used for cell line verification. Real-time RT-PCR identification of candidate PSMArelated genes (MMP3, CDH6 and MTSS1) was performed in 85 clinical prostate samples including 12 normal prostate, 26 benign prostatic hypertrophy (BPH) and 47 prostate cancer samples. (A) MMP3 was expressed at a higher level in the PSMA-negative PC-3 and DU145 cells than in the PSMA-positive LNCap and 22RV1 cells; CDH6 and MTSS1 expression levels were higher in PC-3 cells when compared with the other 3 cell lines. "P<0.05. (B) MMP3, MTSS1, CDH6 and PSMA gene expression levels in clinical tissue samples. Tissues were grouped according to disease state as shown on the x-axis. MMP3, CDH6, MTSS1 and PSMA gene mRNA expression levels are shown on the y-axis. ${ }^{*} \mathrm{P}<0.05$. Each black dot represents a sample data value. Scale bar, median value.

contrast, the expression trend of CDH6 and MTSS1 in the PSMA-negative DU145 cells was different from that in the PC-3 cells

The results of real-time RT-PCR identification in clinical samples showed that the relative MMP3, CDH6 and MTSS1 expression level in normal prostate, BPH and PCa tissues decreased with the development of prostate cancer, and showed a contrary trend with the expression level of PSMA. Expression levels of MMP3, MTSS1 and CDH6 were significantly lower in PCa compared with BPH and normal prostate, respectively $(\mathrm{P}<0.05)$. Correlation analysis demonstrated a negative correlation between the MMP3, MTSS1 and CDH6 mRNA expression and Gleason score (for MMP3, $r=-0.5826$, $\mathrm{P}<0.0001$; for MTSS1, $\mathrm{r}=-0.4727, \mathrm{P}<0.0001$; for $\mathrm{CDH6}$, $\mathrm{r}=-0.4774, \mathrm{P}<0.0001$; Fig. 3B). Negative correlation was also observed between the levels of PSMA and MMP3, MTSS1 and CDH6, indicating that these genes may be involved in the regulation of prostate cancer metastasis mediated by PSMA (for MMP3, $r=-0.6811, \mathrm{P}<0.05$; for $\mathrm{CDH} 6, \mathrm{r}=-0.5430, \mathrm{P}<0.05$; for MTSS1, r=-0.5540, P<0.05) (Fig. 3B).

\section{Discussion}

Prostate cancer metastasis is a complicated and continuous process, which includes tumor cell shedding, invasion, migration, adhesion and proliferation, a process that involves many factors $(20,21)$. Many tumor metastasis-related genes that can promote the tumor metastasis process have been 
reported $(20,21)$, but few tumor metastasis-related genes in prostate cancer have been identified. Therefore, further study of metastasis-related genes is needed to understand the molecular mechanisms of PCa metastasis. In our previous studies, we found that PSMA negatively regulates the process of metastasis in $\mathrm{PCa}$ (12), and blocking of PSMA promotes the invasion and migration of LNCap cells (12), yet the underlying mechanism is still unknown. Therefore, to identify the metastasis-related genes possibly regulated by PSMA, in the present study, we screened 84 metastasis-related genes by tumor metastasis PCR array analysis. The differentially expressed genes between LNCap and PSMA-silenced LNCap cells, and between PSMA-positive LNCap cells and PSMA-negative PC-3 cells were analyzed, and genes possibly related to PSMA were screened by comparison of the common differentially expressed genes in both the si-PSMA group and the PSMA(-) group.

Tumor metastasis PCR array analysis was chosen for the screening of PSMA-related tumor metastasis-related genes. This functional classification gene chip can detect a large number of genes in a parallel analysis, has the characteristics of high-throughput, is fast, accurate and sensitive, and shows superiority in the analysis of disease-related genes and therefore is widely used in oncobiology studies. The 84 genes selected for this array encode many classes of protein factors that are related to tumor metastasis. The results showed that when compared to the PSMA-positive LNCap cells, there were 41 genes upregulated and 15 genes downregulated in the PSMA-negative PC-3 cells. To further identify the significant screened genes that were related to PSMA, we performed gene silencing in PSMA-expressing LNCap cells by siRNA, to ascertain how the PSMA blockage can affect the tumor-metastasis gene expression. The results showed that expression of 14 genes was significantly altered in the si-PSMA LNCap cells, among which 10 genes were upregulated and 4 genes were downregulated. By comparison of the differentially expressed genes between the two groups, we found that 6 genes (IL18, MTSS1, MMP13, ITGA7, MMP3 and CDH6) were upregulated and 2 genes (MDM2 and MMP2) were downregulated in both the PSMA-negative PC-3 cells and the si-PSMA group, suggesting that these genes may be involved in the regulation of prostate cancer cell migration and invasiveness mediated by PSMA. Among the 8 screened PSMA-related genes, CDH6, MDM2 and MMPs were previously reported to be involved in PCa metastasis $(13,22,23)$, whereas other genes found in the present study have not yet been reported.

IL-18 is a cytokine that mediates the immune response and we deduced that its upregulation is very likely to be related to tumor immunity. MTSS1 (metastasis tumor suppressor-1) is a novel putative metastasis-suppressor gene, and its three alternatively splice variants have been confirmed to be downregulated in PCa cell lines and clinical samples, and may contribute to tumor growth and development, as well as metastasis (24). CDH6 (cadherin-6) is a calcium-dependent transmembrane glycoprotein, highly expressed in liver and kidney cancer, and plays an important role in the process of cancer metastasis and invasion. Yet, to date, it has not been reported in PCa (25). MMP2 and MMP3 gene expression levels and enzyme activity were found to be elevated in brain metastases of breast cancer (26). MMP13 also belongs to the
MMP family, and high expression of MMP13 may be an important target for breast cancer (27). MDM2 gene expression is increased in prostate cancer (19), and its function and pathway have been elucidated (22). ITGA7 belongs to the integrin $\alpha$ chain family (28), and has been shown to interact with FHL2 and FHL3 (29). It has also been reported that ITGA7 interacts with high temperature requirement A2 (HtrA2) to induce cell death in human PCa cell lines PC-3 and DU145 (30). Based on the previous reports of these genes, MMP3, MTSS1 and CDH6 were chosen for further verification in prostate cancer cell lines and clinical samples.

Real-time PCR identification results showed that, consistent with the results of gene chip analysis based on LNCap cells and PC-3 cells, MMP3 was expressed at a higher level in PC-3 and DU145 than in LNCap and 22RV1 cells $(\mathrm{P}<0.05)$. Expression levels of CDH6 and MTSS1 were upregulated in PC-3 cells when compared to LNCap, 22RV1 and DU145 cells. Differential expression results of MMP3, MTSS1 and CDH6 shown in tumor metastasis PCR array analysis were confirmed by real-time PCR in the PSMA positive LNCap and 22RV1 cells, and PSMA-negative PC-3 cells, while in DU145, the expression level of CDH6 and MTSS1 was different from that in the PSMA-negative PC-3 cells. Since DU145 was derived from metastatic prostate cancer cells, its metastasis gene expression profile may be different. The underlying mechanism needs further study.

To further identify the expression of these genes in clinical prostate tissues, we detected their expression level in normal prostate, BPH and PCa tissues by real-time PCR. We found that expression levels of MMP3, CDH6 and MTSS1 genes were high in normal prostate tissues, relatively high in BPH tissues, but significantly decreased in PCa samples, and moreover, their expression decreased with the Gleason score and showed high correlation to tumors. It was noteworthy that the expression of these genes showed a negative correlation with PSMA expression level, which suggests that MMP3, CDH6 and MTSS1 may be involved in the regulatory mechanism of tumor metastasis by PSMA, and may also have potential significance as indicators of malignant potential of PCa. Thus, we deduced that PSMA may suppress the migration and invasiveness of PCa cells through directly or indirectly interacting with MMP3, CDH6 and MTSS1. These results may provide important insights into the molecular mechanism of $\mathrm{PCa}$ metastasis. However, further studies are needed to elucidate the possible pathway by which PSMA regulates PCa metastasis and how it interacts with these molecules.

Genes showing discordant change in the PSMA(-) and the si-PSMA groups were noted. CCL7 and ITGB3 genes were upregulated in the si-PSMA LNCap cells, while the expression in PC-3 cells was not significantly changed. IGF1 and CXCL12 genes were highly expressed in the si-PSMA LNCap cells, but at a significantly lower level in the PC-3 cells. Thirteen genes were significantly expressed at a lower level in PC-3 cells but not in the si-PSMA LNCap cells. These genes are reported to play a role in the process of carcinogenesis and tumor metastasis (31-33). Although some of the genes screened in this study were previously reported, e.g., upregulation of CXCL12 is a major mechanism underlying SLUG-mediated migration and invasion of PCa cells (34), most of the differentially expressed genes were reported to be involved in the metastasis 
of a variety of tumors types, but not in $\mathrm{PCa}$. The exact role of these genes in PCa metastasis remains to be explored.

In conclusion, the present study screened differentially expressed tumor metastasis-related genes in LNCap and PC-3 cells and in si-PSMA LNCap and LNCap cells by gene chip technique. Eight genes were screened as PSMA-related by comparison of the common differentially expressed genes, and 3 were further identified in PCa cell lines and clinical samples. These findings provide insight into the regulatory mechanism of the suppression of PCa metastasis by PSMA and the possible interacting proteins. Evidence is provided of the pathway implicated in the suppression of PCa metastasis by PSMA, and provides important clues for further exploration of the molecular mechanisms of PCa metastasis.

\section{Acknowledgements}

The present study was supported by grants from the Natural Science Foundation of China (nos. 81071760, 30772503 and 30371426), and the Natural Science Foundation of Guangdong Province, China (no. 021907).

\section{References}

1. Chang SS: Overview of prostate-specific membrane antigen. Rev Urol 6 (Suppl 10): S13-S18, 2004.

2. Kypta R, Unda M and Carracedo A: Is the bench getting closer to the bedside in the war on cancer? A quick look at prostate cancer. Front Endocrinol 3: 53, 2012.

3. Nelson WG, De Marzo AM and Isaacs WB: Prostate cancer. N Engl J Med 349: 366-381, 2003.

4. Paller CJ, Carducci MA and Philips GK: Management of bone metastases in refractory prostate cancer - role of denosumab. Clin Interv Aging 7: 363-372, 2012.

5. Jemal A, Tiwari RC, Murray T, Ghafoor A, Samuels A, Ward E, Feuer EJ and Thun MJ: Cancer statistics. CA Cancer J Clin 54: 8-29, 2004

6. Germain A, Richardson R, Moul DE, Mammen O, Haas G, Forman SD, Rode N, Begley A and Nofzinger EA: Placebocontrolled comparison of prazosin and cognitive-behavioral treatments for sleep disturbances in US Military Veterans. J Psychosom Res 72: 89-96, 2012.

7. Schroder FH: Progress in understanding androgen-independent prostate cancer (AIPC): a review of potential endocrine-mediated mechanisms. Eur Urol 53: 1129-1137, 2008.

8. Kasper S and Cookson MS: Mechanisms leading to the development of hormone-resistant prostate cancer. Urol Clin North Am 33: 201-210, vii, 2006.

9. Pinto JT, Suffoletto BP, Berzin TM, Qiao CH, Lin S, Tong WP, May F, Mukherjee B and Heston WD: Prostate-specific membrane antigen: a novel folate hydrolase in human prostatic carcinoma cells. Clin Cancer Res 2: 1445-1451, 1996.

10. Robinson MB, Blakely RD, Couto R and Coyle JT: Hydrolysis of the brain dipeptide N-acetyl-L-aspartyl-L-glutamate. Identification and characterization of a novel N-acetylated alphalinked acidic dipeptidase activity from rat brain. J Biol Chem 262: 14498-14506, 1987.

11. Cao KY, Mao XP, Wang DH, Xu L, Yuan GQ, Dai SQ, Zheng BJ and Qiu SP: High expression of PSM-E correlated with tumor grade in prostate cancer: a new alternatively spliced variant of prostate-specific membrane antigen. Prostate 67: 1791-1800, 2007.

12. Cao KY, Xu L, Zhang DM, Zhang XM, Zhang T, He X, Wang Z, Feng FS, Qiu SP and Shen GX: New alternatively spliced variant of prostate-specific membrane antigen PSM-E suppresses the proliferation, migration and invasiveness of prostate cancer cells. Int J Oncol 40: 1977-1985, 2012.

13. Ghosh A and Heston WD: Tumor target prostate specific membrane antigen (PSMA) and its regulation in prostate cancer. J Cell Biochem 91: 528-539, 2004.
14. Frigerio B, Fracasso G, Luison E, Cingarlini S, Mortarino M, Coliva A, Seregni E, Bombardieri E, Zuccolotto G, Rosato A, Colombatti M, Canevari S and Figini M: A single-chain fragment against prostate specific membrane antigen as a tool to build theranostic reagents for prostate cancer. Eur J Cancer, pii: S0959-8049(13)00087-7. doi: 10.1016/j.ejca.2013.01.024., 2013.

15. Zhao LY, Mao XP, Chao KY, Guo SJ and Qiu SP: Prostate-specific membrane antigen can promote in vivo osseous metastasis of prostate cancer cells in mice. Braz J Med Biol Res 45: 737-745, 2012.

16. Mannweiler S, Amersdorfer P, Trajanoski S, Terrett JA, King D and Mehes G: Heterogeneity of prostate-specific membrane antigen (PSMA) expression in prostate carcinoma with distant metastasis. Pathol Oncol Res 15: 167-172, 2009.

17. Ghosh A, Wang X, Klein E and Heston WD: Novel role of prostate-specific membrane antigen in suppressing prostate cancer invasiveness. Cancer Res 65: 727-731, 2005.

18. Xie BX, Zhang H, Wang J, Pang B, Wu RQ, Qian XL, Yu L, Li SH, Shi QG, Huang CF and Zhou JG: Analysis of differentially expressed genes in $\mathrm{LNCaP}$ prostate cancer progression model. J Androl 32: 170-182, 2010.

19. Wang G, Firoz EF, Rose A, Blochin E, Christos P, Pollens D, Mazumdar M, Gerald W, Oddoux C, Lee P and Osman I: MDM2 expression and regulation in prostate cancer racial disparity. Int $\mathrm{J}$ Clin Exp Pathol 2: 353-360, 2009.

20. Kohn EC: Invasion and metastasis: biology and clinical potential. Pharmacol Ther 52: 235-244, 1991.

21. Duffy MJ, McGowan PM and Gallagher WM: Cancer invasion and metastasis: changing views. J Pathol 214: 283-293, 2008.

22. Kong L, Yuan Q, Zhu H, Li Y, Guo Q, Wang Q, Bi X and Gao X: The suppression of prostate LNCaP cancer cells growth by Selenium nanoparticles through Akt/Mdm2/AR controlled apoptosis. Biomaterials 32: 6515-6522, 2011.

23. Roomi MW, Monterrey JC, Kalinovsky T, Rath $M$ and Niedzwiecki A: Inhibition of invasion and MMPs by a nutrient mixture in human cancer cell lines: a correlation study. Exp Oncol 32: 243-248, 2011.

24. Loberg RD, Neeley CK, Adam-Day LL, Fridman Y, St John LN, Nixdorf S, Jackson P, Kalikin LM and Pienta KJ: Differential expression analysis of MIM (MTSS1) splice variants and a functional role of MIM in prostate cancer cell biology. Int J Oncol 26: 1699-1705, 2005.

25. Inoue $\mathrm{T}$, Inoue $\mathrm{YU}$, Asami J, Izumi $\mathrm{H}$, Nakamura $\mathrm{S}$ and Krumlauf R: Analysis of mouse Cdh6 gene regulation by transgenesis of modified bacterial artificial chromosomes. Dev Biol 315: 506-520, 2008.

26. Mendes O, Kim HT and Stoica G: Expression of MMP2, MMP9 and MMP3 in breast cancer brain metastasis in a rat model. Clin Exp Metastasis 22: 237-246, 2005

27. Chang HJ, Yang MJ, Yang YH, Hou MF, Hsueh EJ and Lin SR: MMP13 is potentially a new tumor marker for breast cancer diagnosis. Oncol Rep 22: 1119-1127, 2009.

28. Wang W, Wu W, Desai T, Ward DC and Kaufman SJ: Localization of the alpha 7 integrin gene (ITGA7) on human chromosome 12q13: clustering of integrin and Hox genes implies parallel evolution of these gene families. Genomics 26: 568-570, 1995.

29. Samson T, Smyth N, Janetzky S, Wendler O, Müller JM, Schüle R, von der Mark $\mathrm{H}$, von der Mark K and Wixler V: The LIM-only proteins $\mathrm{FHL}_{2}$ and $\mathrm{FHL}_{3}$ interact with $\alpha$ - and $\beta$-subunits of the muscle $\alpha_{7} \beta_{1}$ integrin receptor. J Biol Chem 279: 28641-28652, 2004.

30. Zhu ZH, Yu YP, Zheng ZL, Song Y, Xiang GS, Nelson J, Michalopoulos $\mathrm{G}$ and Luo $\mathrm{JH}$ : Integrin alpha 7 interacts with high temperature requirement A2 (HtrA2) to induce prostate cancer cell death. Am J Pathol 177: 1176-1186, 2010.

31. Mazzoccoli G, Sothern RB, Pazienza V, Piepoli A, Muscarella LA, Giuliani F and Tarquini R: Circadian aspects of growth hormone-insulin-like growth factor axis function in patients with lung cancer. Clin Lung Cancer 13: 68-74, 2011.

32. Montagnani Marelli M, Moretti RM, Procacci P, Motta M and Limonta P: Insulin-like growth factor-I promotes migration in human androgen-independent prostate cancer cells via the $\alpha_{v} \beta_{3}$ integrin and PI3-K/Akt signaling. Int J Oncol 28: 723-730, 2006.

33. Nickerson T, Chang F, Lorimer D, Smeekens SP, Sawyers CL and Pollak M: In vivo progression of LAPC-9 and LNCaP prostate cancer models to androgen independence is associated with increased expression of insulin-like growth factor I (IGF-I) and IGF-I receptor (IGF-IR). Cancer Res 61: 6276-6280, 2001.

34. Uygur B and Wu WS: SLUG promotes prostate cancer cell migration and invasion via CXCR4/CXCL12 axis. Mol Cancer 10: 139,2011 